\title{
Application of Variation Iteration Method to Material Transport Problems in Finite Membranes
}

\author{
K.Venugopal \\ Department of Mathematics \\ J.J College of Engg \& Tech \\ Tiruchirapalli-620 009 \\ Tamilnadu. India
}

\author{
L.Rajendran \\ Department of Mathematics \\ The Madura College \\ Madurai-625011 \\ Tamilnadu. India
}

\begin{abstract}
A mathematical model of the transport material through a membrane of finite thickness via the process of diffusion has been developed. We may consider a membrane in between a donor and a receptor compartment. the cause of an externally applied electric field and concurrent first-order chemical reaction of the diffusion species with sites in the membrane on the diffusion state is examined via the formulation of a time dependant differential equation and its subsequent solution by variational iteration method(VIM). A simple closed form of analytical expression for the concentration profile is derived and compared with the previous results and found to be in good agreement.
\end{abstract}

Keywords: Diffusion, membranes, Diffusion équations. Active diffusion, Iontophoresis.

\section{INTRODUCTION}

The study of material transport through diffusion in regions of finite spatial extent is a subject of much more interest. For many more years before these study relates theoretical aspects, by mathematical modeling, the reference produced by Carssaw and Jaeger[1] and Crank [2] . The analyses of bounded diffusion process in which the diffusing materials, subject in the electric fields and can undergo chemical reactions are most interesting area to find a new solution. It can be useful for chemical reaction with sites located in diffusion medium. The differential equation is then solved to get a closed form expression for the concentration profile of the diffusion as a function and a time. In particular, for simple passive diffusion through the membrane, the lag time $t$, may be used to obtain an estimate of the diffusion co-efficient $\mathrm{D}$ of the transported species, through the expression, $t_{L}=L_{2} / 6 D$

The previous workers Ludolph and co-workers[3] were interested to calculate the lag-time expected far bounded diffusion coupled with chemical reaction, which yields a solution for equilibrium constant relating free and boundary penetrant. After that Keister and Kasting[5] modeled electric field enhanced active diffusion within a finite membrane by a separate variable method. M.E. Lyans[7] and his co-workers has arrived at solution using Laplace transform Technique.

In this paper we are present an alternative analysis of bounded diffusion with concurrent chemical reaction and electro migration to obtain the closed form of analytical expression for the concentration profile of the penetrant and total quantity of diffusion exiting the membrane, as a function of time.

\section{MATHEMATICAL} FORMULATION AND DESCRIPTION OF THE PHYSICAL SYSTEM

The mathematical model used to determine the following experimental arrangement. Let us consider a thin homogenous membrane of finite thickness L, which separate the two bulk volumes which is clearly indicated diagrammatically in fig(i). Let us assume that the diffusion of penetrant is planar. Hence the special variable is defined when the range $0 \leq x \leq L$.

The region $\chi=0$ is assigned to the donor compartment, while $\chi=1$ is assigned to the acceptor compartment. During this process we assume that membrane is subjected to a constant electric field. In addition to that the diffusing penetrant reacts within the membranes to a first order kinetic expression with rate constant $\mathrm{k}$.

At time $\mathrm{t}=0$, the face of the membrane adjacent to the donor region is exposed to a constant concentration $\mathrm{C}_{0}$ while other faces which is in contact with acceptor region is maintained at zero concentration. Further we assume that the solution in both region ie donor as well as acceptor compartments are well stirred, besides the receiver solution acts as an infinite sink and the donor acts as an infinite source.

The mathematical description from the above problem, which involves diffusion equation of the following type.

Let us consider the time dependant diffusion equation of the following type

$$
\frac{\partial u}{\partial \tau}=\frac{\partial^{2} u}{\partial^{2} \chi}-\beta \frac{\partial u}{\partial \chi}-\gamma u, 0 \leq u \leq 1 ; 0 \leq \chi \leq 1
$$

This expression is obtainable in non dimensional form using the following normalized parameter.

$u=\frac{c}{k c_{0}}, \chi=\frac{x}{L}, \gamma=\frac{k L^{2}}{D}=\frac{j_{R}}{j_{D}}, \beta=\frac{\mu E L}{D}=\frac{j_{M}}{j_{D}}, \tau=\frac{D t}{L^{2}}$ 
where $u$ represents a non-dimensional penetrant concentration at any point, $\chi$ represents normalized distance variable scaled to the total thickness $\mathrm{L}$ of the membrane, $\tau$ represents Normalized time parameter, $\beta$ denotes the diffusion migration parameters and $\gamma$ denotes diffusion reaction parameter. Ratio $\psi / \beta$ compare the rate of penetrant species reaction at a site in the membrane to the rate of electro migration of penetrant species within the membrane. The diffusion reaction parameter $\gamma$ is defined as the ratio of the flux due to the chemical reaction to the flux arising from the species diffusion through the membrane. By assuming the form

$$
u(\chi, \tau)=\exp [\xi x] \exp [-\zeta \tau] \omega(\chi, \tau)
$$

The eqn.(1) becomes

$$
\frac{\partial \omega}{\partial \tau}=\frac{\partial^{2} \omega}{\partial \chi^{2}}
$$

The problem is defined with the following initial and boundary conditions.

$$
\begin{aligned}
& \omega(\chi, 0)=0 \\
& \omega(0 . \tau)=\exp (\varsigma \tau) \\
& \omega(1, \tau)=0
\end{aligned}
$$

Where

$$
\xi=\frac{\beta}{2}, \varsigma=\gamma+\xi^{2}
$$

First we solve the equation(5) and extend the result how the more complex situation of diffusion coupled with concurrent electro migration and chemical reaction which is governed by differential equation given by equation(1).

In both cases we utilize the technique of Variational iteration methods (VIM's), which is solution technique of choice when bounded diffusion problems are examined.

\section{BASIC CONCEPTS IN THE VARIATIONAL ITERATION METHOD AND SOLUTION OF THE EQUATION}

To illustrate the basic concepts of variational iteration method (VIM), we consider the following non-linear partial differential equation

$$
L[\omega(\chi, \tau)]+N[\omega(\chi, \tau)]=g(\chi, \tau)
$$

where $L$ is a linear operator, $N$ is a nonlinear operator, and $g(x)$ is a given continuous function. According to the variational iteration method, we can construct a correct functional as follows:

$$
\omega_{n+1}(x)=\omega_{n}(x)+\int_{0}^{x} \lambda\left[L\left[\omega_{n}(\tau)\right]+N\left[\tilde{\omega_{n}}(\tau)\right]-g(\tau)\right] d \tau(7)
$$

where $\lambda$ is a general Lagrange multiplier which can be identified optimally via variational theory, $\omega_{n}$ is the $\mathrm{n}^{\text {th }}$ approximate solution, and $\tilde{\omega_{n}}$ denotes a restricted variation, i.e., $\delta \tilde{\omega}_{n}=0$. In this method, a trail function (an initial solution) is chosen with some unknown parameter, which is identified after a few iteration according to the given boundary conditions .Using above variation iteration method we can write the correction functional of eqn. (4) as follows

$\omega_{n+1}(\chi, \tau)=\omega_{n}(x, \tau)+\int_{0}^{x} \lambda_{1}[\overbrace{\omega_{n}^{\prime \prime}(\xi)}^{\tau}-\omega_{\chi \chi}] d \xi$

Taking variation with respect to the independent variable $\omega_{n}$, weget

$$
\delta \omega_{n+1}(\chi, \tau)=\delta \omega_{n}(\chi, \tau)+\delta \int_{0}^{x} \lambda_{1}\left[-\omega_{\chi \chi}(\xi)\right] d \xi
$$

where $\lambda$ is general Lagrangian multipliers, consider restricted variations i.e $\delta \omega_{n}=0$ and making the above correction functional (9) stationary, noticing that $\delta \omega_{n}(0)=0$,

$$
\begin{aligned}
& \delta \omega_{n}: 1+\left.2 \lambda^{1}(\xi)\right|_{\xi=x}=0 \\
& \delta \omega_{n}^{\prime}:\left.\lambda(\xi)\right|_{\xi=x}=0 \\
& \delta \omega_{n}:\left.\lambda^{\prime \prime}(\xi)\right|_{\xi=x}=0-
\end{aligned}
$$

The above equation is called Lagrange-Euler equation. The Lagrange multiplier, can be identified as

$$
\lambda(\xi)=\chi-\xi
$$

Substituting the Lagrangian multipliers and $\mathrm{n}=0$ in the iteration formula (eqn (9) ) we obtain,

$$
\begin{aligned}
& \omega_{1}(\chi, \tau)=u_{0}(\chi \cdot \tau)+\int_{0}^{x}(\chi-\xi)\left[\omega_{\tau}(\xi)-\omega_{0 \chi \chi}(\xi)\right] d \xi \\
& \omega_{1}(\chi, \tau)=e^{\zeta \tau}-e^{\chi \tau}\left[\frac{\chi^{2}}{\tau}+\frac{(\chi \tau+2) \chi}{\tau^{2}}\right]--(15)
\end{aligned}
$$

Using the equation (3) we obtain

$$
\begin{aligned}
& u_{1}(\chi, \tau)=\exp \llbracket] \exp \Leftarrow \zeta \tau] \omega(\chi, \tau) \\
&\left.=\exp \notin] \exp [\zeta \tau] e^{\zeta \tau}-e^{\chi \tau}\left[\frac{\chi^{2}}{\tau}+\frac{(\chi \tau+2) \chi}{\tau^{2}}\right]\right]
\end{aligned}
$$




\section{FIRST ORDER CHEMICAL REACTION IN A FINITE MEMBRANE ASSISTED WITH ELECTRIC FIELD}

Here we will give solution to equation (1) which describes substrate transport and reaction within a free-standing electronically conducting polymer membrane in which diffusing substrate reacts, which is typically corresponds to iontophoretic transport of charged drug species across a membrane barrier in which the drug can be metabolized through first order kinetics. Thus it calibrates the electric field assisted by diffusion with concurrent first order reaction kinetics in a finite membrane.

The parameters $\beta \& \gamma$ are both zero and simple passive diffusion pertains. Normalized concentration profile for penetrant can be obtained. These profiles may be used in three dimensional formats for the ease of representations. For example the normalized concentration profile ' $u$ ' varies with $\beta$ at different values of normalized time $\tau$ ranging from $\tau=0.01$ to steady state, when the parameter $\psi$ is zero.

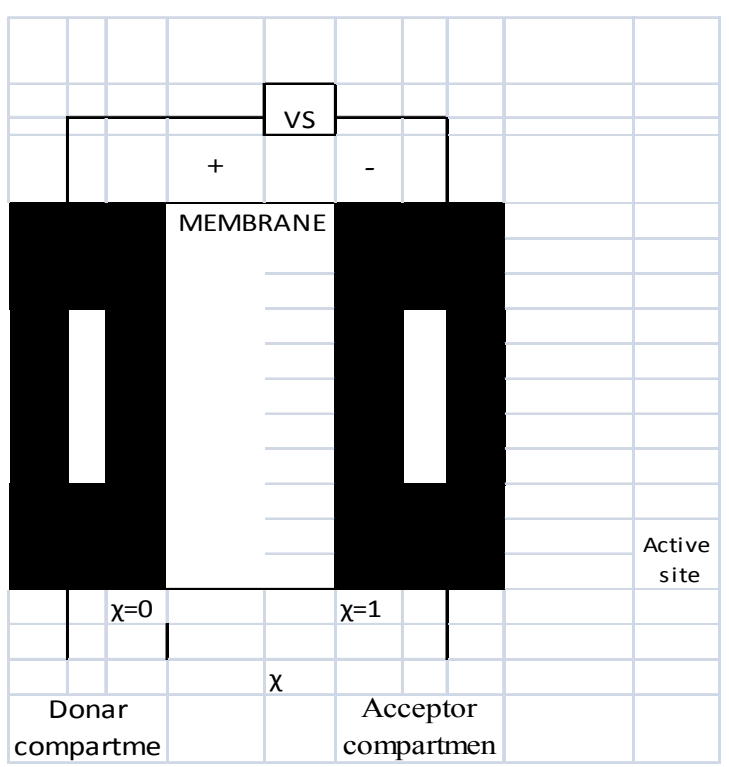

Figure 1. Schematic representation of free standing membrane of finite thickness $L$, containing immobilized active binding reaction sites. The penetrant species passes through the membrane from a donor to a receptor compartment. A uniform electric field is present in the membrane which can facilitate transport of penetrant

\section{CONCLUSIONS}

In this paper, He's varitationals iteration method has been successfully applied to find out the solution of non linear boundary value problem with serial linear and nonlinear equations. In our work, we use the Maple package to calculate the functions obtained from the variational iterations method. Some of the advantages of a VIM are that the initial solutions can be freely chosen with some unknown parameters and that we can easily achieve the unknown parameters in the initials solutions. This paper applies the variational iteration method to nonlinear wave equation, the solution process is simple but effective result is of high accuracy. Compared with HAM method proposed in [8], the present method has many advantages; the HAM requires complex calculation of suitable choice of leads to ideal results or results in wrong solutions. The present paper completely overcome the difficulty arising in HAM . An interesting point about VIM is that with fewest number of iterations or even in some cases, once, it can converge to correct results.

\section{ACKNOWLEDGEMENT}

The author wish to thank the Management of J.J. college of Engineering and Technology, Tiruchirapalli 620 009, for providing the opportunity to my research work and continuous constant encouragement to brink out this article in successful manner.

\section{REFERENCES}

[1] Carslaw HS, Jaeger JC(1997) Conduction of heat in solids, $2^{\text {nd }}$ edn, Clarendon Press, Oxford

[2] Crank J(1998) The mathematics of diffusion , $2^{\text {nd }}$ edn, Clarendon Press, Oxford.

[3] Ludolph RA, Veith WR, Friseh HL(1979) J phys Chem $83: 2793$

[4] Leypoldt JK, Gough DA(1980) J phys Chem 84:1058

[5] Keister JC, Kasting GB(1986) J Membr Sei 29:155

[6] Chen JS, Rosenberger F(1991) J phys Chem 95:10164

[7] M.E.Lyans , Judith Murphy, Serge Rebouillat J Solid State ElectroChemistry (2000)

[8] Fakhari, A., Domairry, G.,Ebrahimpour: Approximate explicit solutions of nonlinear BBMB equations by Homotopy analysis Method and and comparison with exact solutions. Phys.Lett.A 368, 64-68(2007)

[9] Churchill RV(1972) Operational mathematics. $3^{\text {rd }}$ edn. McGraw-Jill, New York.p 464

[10] Spanier J.Oldham KB(1987)An atlas of functions, Hemisphere ,New yoark. pp390-391.

[11] He J.H. J.Comput Appl.Math.207,3(2007) and reference therein.

[12] He.J.H., Abdou, Chaos Solutions Fracticals 34(5), 1430 (2007)

[13] He.J.H. Int. J. Nonlinear Sci. Numer.Simulat.2(4),230(1997)

[14] He J.H. J.Comput.Appl.Math. 207, I(2007)

[15] Hossein Jafari et.al Applied Mathe. Science 2(10),471477(2008) 